\title{
Exposure to artemisinin at the trophozoite stage increases sexual conversion rates in the malaria parasite Plasmodium falciparum
}

Harvie P. Portugaliza ${ }^{1,2,3}$, Shinya Miyazaki ${ }^{4,},{ }^{\star}$ Fiona J.A. Geurten ${ }^{4}$, Christopher Pell $^{3,5}$, Anna Rosanas-Urgell ${ }^{2}$, Chris J. Janse ${ }^{4}$ and Alfred Cortés ${ }^{1,6, \#}$

${ }^{1}$ ISGlobal, Hospital Clinic - Universitat de Barcelona, Barcelona, Catalonia, Spain

${ }^{2}$ Department of Biomedical Sciences, Institute of Tropical Medicine, Antwerp, Belgium

${ }^{3}$ Department of Global Health, Amsterdam University Medical Centers, location Academic Medical Center, University of Amsterdam, Amsterdam, The Netherlands

${ }^{4}$ Department of Parasitology, Leiden University Medical Center, Leiden, The

Netherlands

${ }^{5}$ Amsterdam Institute for Global Health and Development (AIGHD), Amsterdam, The Netherlands

${ }^{6}$ ICREA, Barcelona, Catalonia, Spain

"Current address: Department of Cellular Architecture Studies, Institute of Tropical Medicine (NEKKEN), Nagasaki University, Nagasaki, Japan

\#Correspondence: alfred.cortes@isglobal.org (Alfred Cortés) 


\section{ABSTRACT}

Malaria transmission is dependent on formation of gametocytes in the human blood. The sexual conversion rate, the proportion of asexual parasites that convert into 30 gametocytes at each multiplication cycle, is variable and reflects the relative parasite investment between transmission and maintaining the infection. The impact of environmental factors such as drugs on sexual conversion rates is not well understood. We developed a robust assay using gametocyte-reporter parasite lines to accurately measure the impact of drugs on conversion rates, independently from their gametocytocidal activity. We found that exposure to subcurative doses of the frontline antimalarial drug dihydroartemisinin (DHA) at the trophozoite stage resulted in a 4 -fold increase in sexual conversion. In contrast, no increase was observed when ring stages were exposed or in cultures in which sexual conversion was stimulated by choline depletion. Our results reveal a complex relationship between antimalarial drugs and sexual conversion, with potential public health implications. 


\section{INTRODUCTION}

Plasmodium falciparum is responsible for the most severe forms of human malaria.

Repeated rounds of its $\sim 48 \mathrm{~h}$ intraerythrocytic asexual replication cycle result in an exponential increase in parasite numbers and are responsible for all clinical symptoms of malaria. At each round of replication, a small subset of the parasites commits to differentiation into non-replicative sexual forms termed gametocytes, which are the only form transmissible to a mosquito vector. Sexual commitment is marked by epigenetic activation of the master regulator PfAP2-G, a transcription factor of the ApiAP2 family (Josling et al, 2020; Kafsack et al, 2014; Llorà-Batlle et al, 2020; Poran et al, 2017). This is followed by sexual conversion, which according to our recently proposed definitions (Bancells et al, 2019) is marked by expression of gametocyte-specific proteins absent from any replicating blood stages. After sexual conversion, parasites at the sexual ring stage develop through gametocyte stages I to $\mathrm{V}$ in a maturation process that lasts for $\sim 10$ days (Josling et al, 2018; Ngotho et al, 2019). While immature gametocytes are sequestered in organs such as the bone marrow (Venugopal et al, 2020), mature gametocytes (stage V) are released into the circulation, where they are infectious to mosquitoes for several days or even weeks (Cao et al, 2019). To eliminate malaria, which the World Health Organization has adopted as a global goal, it is necessary to block transmission, as well as killing asexual parasites to cure patients (World_Health_Organization, 2017).

To secure within-host survival and between-host transmission, the proportion of parasites that convert into sexual forms at each replicative cycle, termed sexual conversion rate, is variable and tightly regulated. In human infections, gametocyte densities are always much lower than asexual parasite densities, and basal $P$. falciparum sexual conversion rates in vivo are estimated to be $\sim 1 \%$ (Cao et al, 2019; Eichner et al, 2001). This reveals a reproductive restraint for which multiple alternative hypotheses have been proposed (McKenzie \& Bossert, 1998; Mideo \& Day, 2008; Taylor \& Read, 1997). Whatever the reason for the low levels of sexual conversion, multiple observations suggest that malaria parasites can respond to changes in the conditions of their environment by adjusting the trade-off between transmission and within-host survival. From an evolutionary perspective, the ability to adjust sexual conversion rates depending on the host conditions would be clearly 
falciparum, several specific conditions have been shown to increase sexual conversion rates, and exposure to stress in general was proposed to enhance sexual conversion (Baker, 2010; Bousema \& Drakeley, 2011; Dyer \& Day, 2000; Josling et al, 2018). The most commonly used method to enhance sexual conversion and obtain large numbers of gametocytes in vitro relies on overgrowing blood stage cultures (the "crash method") (Delves et al, 2016) and/or maintaining the cultures with parasite-conditioned (spent) medium (Brancucci et al, 2015; Fivelman et al, 2007). Recent research has established that depletion of the human serum lipid lysophosphatidylcholine (LysoPC) underlies the stimulation of sexual conversion by high asexual parasitemia or spent medium, providing the first mechanistic insight into how environmental conditions can influence the rate of sexual conversion (Brancucci et al, 2018; Brancucci et al, 2017). Low plasma LysoPC levels were also associated with increased sexual conversion rates in human infections (Usui et al, 2019). Depletion of LysoPC or choline, a downstream metabolite in the same metabolic pathway, have now been used by several groups to stimulate sexual conversion under culture conditions (Brancucci et al, 2017; Filarsky et al, 2018; Portugaliza et al, 2019).

Artemisinin and its derivatives (collectively referred to as ARTs) are potent antimalarial drugs that rapidly kill asexual parasites. After activation by cleavage of their endoperoxide bond by haemoglobin degradation products, ARTs produce reactive oxygen species and free radicals that result in widespread damage in parasite proteins and lipids. However, because ARTs have a very short elimination half-life in the human circulation ( 1-3 h), their application as monotherapy was discontinued to avoid infection recrudescence and development of drug resistance.

100 Artemisinin-based combination therapies (ACTs), consisting of ART and a longacting partner drug, are the current frontline treatment for uncomplicated as well as severe malaria cases (Blasco et al, 2017; de Vries \& Dien, 1996; Haldar et al, 2018; Talman et al, 2019). Resistance to ARTs has emerged in South-East Asia in the form of delayed parasite clearance (Dondorp et al, 2009). ART resistance is associated with mutations in the PfKelch13 protein (Ariey et al, 2014) that prevent haemoglobin degradation in early ring-stage parasites. This in turn prevents ART activation, resulting in resistance of early rings to the drug (Birnbaum et al, 2020; Yang et al, 2019). Nowadays, ART resistance is frequently accompanied by 
simultaneous resistance to partner drugs such as mefloquine, piperaquine or amodiaquine, resulting in high rates of treatment failure and limiting treatment options (Mairet-Khedim et al, 2020; Phyo et al, 2016; van der Pluijm et al, 2019).

Treatment with antimalarial drugs such as chloroquine (CQ) or sulfadoxinepyrimethamine is usually associated with increased gametocytemia (density of gametocytes in the blood) on the days following drug administration, whereas

115 treatment with ACTs results in reduced gametocytemia and transmission to mosquitoes (Ippolito et al, 2017; Okell et al, 2008; Price et al, 1996; Sawa et al, 2013; von Seidlein et al, 2001; WWARN_Gametocyte_Study_Group, 2016). Despite the efficacy of ACTs in reducing gametocytemia, successfully treated patients can remain infectious for several days and contribute to transmission (Bousema et al, 120 2006; Bousema et al, 2010; Karl et al, 2015; Targett et al, 2001). The higher capacity of ACTs to reduce gametocytemia compared to other drugs is attributable to several factors: i) faster killing of asexual parasites, which prevents the formation of new gametocytes; ii) more efficient killing of immature gametocytes; iii) partial clearance of mature gametocytes, which are insensitive to most other clinically relevant drugs (Adjalley et al, 2011; Chotivanich et al, 2006; Plouffe et al, 2016).

Although it has been proposed that the increase of gametocytemia observed after treatment with some drugs may reflect stimulation of sexual conversion, there is no direct linear relationship between conversion rates and the prevalence and density of circulating gametocytes (Carter et al, 2013; Koepfli \& Yan, 2018; Reece \& Schneider, 2018). The dynamics of circulating gametocyte densities after treatment can be explained without invoking an adjustment of sexual conversion rates: first, gametocytes are sequestered away from the circulation until $\sim 10$ days after sexual conversion, implying that the peaks of gametocytemia observed after treatment with some drugs (within less than 10 days) may reflect the dynamics of asexual parasite growth before treatment, rather than post-treatment changes in sexual conversion. Second, the effects of the drugs on sexual conversion rates in human infections cannot be disentangled from other drug-mediated actions such as the release of sequestered parasites or gametocyte clearance (Babiker et al, 2008; Bousema \& Drakeley, 2011; Butcher, 1997; Koepfli \& Yan, 2018). 
To directly address the effect of drug treatment on sexual conversion, a small number of studies have used $P$. falciparum in vitro cultures, yielding inconsistent results. While some studies reported increased sexual conversion upon exposure to specific doses of drugs such as CQ or ART (Buckling et al, 1999b; Peatey et al, 2009; Rajapandi, 2019), others did not observe this effect with ART (Brancucci et al,

145 2015), or reported reduced sexual conversion upon exposure to low doses of CQ or pyrimethamine (Reece et al, 2010). Although the discrepancies may reflect methodological differences between these studies and limited accuracy in determining sexual conversion rates, the divergent conclusions also suggest a complex scenario in which conditions such as the specific drug used, the parasite stage at the time of exposure, and drug concentration may determine the effect of treatment on sexual conversion.

Given the widespread use of ACTs for malaria treatment and in mass drug administration campaigns aimed at malaria elimination, understanding the impact of ARTs on sexual conversion is an urgent research priority. Here, we developed a robust assay based on recently described gametocyte-reporter parasite lines (Portugaliza et al, 2019) to accurately measure the impact of drugs on sexual conversion rates, independently from their gametocytocidal activity. Using this assay, we tested the effect of exposing parasites to dihydroartemisinin (DHA, the active metabolite of all ARTs) and CQ at different stages and under different metabolic conditions, to provide an accurate and comprehensive description of the direct effect of these drugs on sexual conversion rates.

\section{RESULTS}

\section{Exposure to DHA at the trophozoite stage enhances sexual conversion}

165 To examine the effect of ARTs on $P$. falciparum sexual conversion, we administered a $3 \mathrm{~h}$ pulse of DHA to synchronous cultures of the NF54-gexp02-Tom reporter line. This parasite line expresses the fluorescent reporter tdTomato under the control of the promoter of the sexual stage-specific gene gexp02 (PF3D7_1102500), which allows accurate flow cytometry-based detection of very early gametocytes within a 170 few hours after sexual conversion (Portugaliza et al, 2019). The short drug pulse mimics the short plasma half-life of ARTs (de Vries \& Dien, 1996). Cultures were 
regularly maintained in choline-containing culture medium (Albumax-based medium with a supplement of choline) to mimic the repression of sexual conversion by healthy human serum, and choline was either maintained or removed during the experiment to repress or stimulate sexual conversion (Brancucci et al, 2017; Filarsky et al, 2018). The DHA pulse was administered at the trophozoite (Fig. 1A) or the ring stage (Fig. 2A), using subcurative DHA concentrations (5 and $10 \mathrm{nM}$ ) that resulted in a reduction of growth of $<40 \%$ and $<25 \%$ in trophozoites and rings, respectively (Fig. $1 \mathrm{~B}$ and $2 \mathrm{~B}$ ). The sexual conversion rate was calculated as the proportion of parasites that developed into gametocytes at the cycle after exposure (i.e., after reinvasion).

In cultures supplemented with choline, the sexual conversion rate increased from $<10 \%$ in control cultures to up to $40 \%$ in cultures exposed to a 5 or $10 \mathrm{nM} \mathrm{DHA}$ pulse at the trophozoite stage (Fig. 1C-D). Importantly, total gametocytemia was also clearly higher in DHA-exposed cultures than in control cultures (Fig. 1E). This result indicates that the increase in the sexual conversion rate is not only attributable to the lower number of asexual parasites after drug treatment, but also to a net increase in the number of gametocytes produced. By contrast, in cultures in which sexual conversion was already stimulated by choline depletion, DHA treatment did not result 190 in a further increase in the sexual conversion rate or in the absolute number of gametocytes (Fig. 1C-E). Similar results were obtained in experiments in which sexual conversion rates were calculated based only on viable parasites as identified by a marker of active mitochondria (Supplementary Fig. 1), using an analogous reporter line generated in the 3D7-E5 genetic background that has lower levels of basal sexual conversion than NF54 (E5-gexp02-Tom line, Supplementary Fig. 2) (Portugaliza et al, 2019), and using a transgenic line with the fluorescent reporter under the control of the etramp10.3 (PF3D7_1016900) gametocyte-specific promoter (NF54-10.3-Tom line) (Portugaliza et al, 2019). Using this latter parasite line, we measured sexual conversion rates by flow cytometry, by immunofluorescence assay (IFA) detecting the Pfs16 (PF3D7_0406200) early gametocyte marker and by light microscopy analysis of Giemsa-stained blood smears. All approaches yielded similar results and confirmed enhanced sexual conversion after exposure of trophozoites to subcurative doses of DHA (Supplementary Fig. 3). 
We also tested the impact on sexual conversion of higher DHA doses up to 30 $\mathrm{nM}$, a concentration that kills $\sim 90 \%$ of the parasites (Supplementary Fig. 4A-B). In choline-supplemented cultures, both sexual conversion rates and total gametocytemia were clearly enhanced upon exposure to DHA concentrations up to $15 \mathrm{nM}$, but the increase was lower upon exposure to higher concentrations. In choline-depleted cultures, $>10 \mathrm{nM}$ DHA resulted in a reduction of sexual conversion

210 compared with no drug controls (Supplementary Fig. 4). However, it is important to note that the determination of sexual conversion rates is less accurate when the majority of the parasites are killed by the drug. Thus, given that maximum induction was observed at $10 \mathrm{nM}$ and the difficulties to estimate sexual conversion accurately in experiments with higher drug doses, we used 5 and $10 \mathrm{nM}$ DHA pulses for the

215 experiments described in the next sections.

Gametocytes of the NF54-gexp02-Tom line produced in cultures treated with $5 \mathrm{nM}$ DHA matured through stages I to $\mathrm{V}$ without any apparent morphological alteration. Furthermore, they were able to exflagellate and to infect mosquitoes (Supplementary Fig. 5).

\section{DHA exposure at the ring stage does not enhance sexual conversion}

A DHA pulse (5 or $10 \mathrm{nM}$ ) at the early ring stage did not enhance sexual conversion. Instead, it resulted in a reduction of sexual conversion and gametocytemia, both in choline-supplemented and choline-depleted NF54-gexp02-Tom cultures (Fig. 2;

225 Supplementary Fig. 6). This unexpected result was confirmed using the NF54-10.3Tom reporter line and the different methods described above to assess sexual conversion (Supplementary Fig. 7). To explore the possibility that DHA exposure at the early ring stage may stimulate immediate sexual conversion via the same cycle conversion (SCC) pathway (Bancells et al, 2019), rather than by the canonical next

230 cycle conversion (NCC) pathway, we assessed the effect of DHA exposure at the ring stage on the level of gametocytes produced within the same cycle of exposure (Fig. 3A). We observed no apparent differences in sexual conversion rates via the SCC route between DHA-exposed cultures and their controls (Fig. 3B-C; Supplementary Fig. 8). Similar results were obtained using the NF54-10.3-Tom reporter line and flow cytometry or light microscopy analysis of Giemsa-stained 
smears to measure sexual conversion by the SCC pathway. However, IFA analysis of this parasite line using anti-Pfs16 antibodies revealed an increase in the proportion of parasites expressing this endogenous protein upon DHA exposure (Supplementary Fig. 9). The significance of this observation remains unclear, but it

240 may indicate a rapid effect of DHA on the expression of some gametocyte specific genes without further sexual development.

These experiments also revealed that choline depletion did not increase sexual conversion via the SCC route (Fig. 3B). This result may be explained by two alternative scenarios: i) conversion via the SCC route is insensitive to choline 245 depletion; ii) ring stages are insensitive to choline depletion. To distinguish between these two possibilities, we assessed sexual conversion via the NCC pathway in cultures in which choline was depleted at different stages of the life cycle (Fig. 4A). We found that choline depletion at the ring stage does not induce sexual conversion, in contrast to depletion at the trophozoite stage (Fig. 4B-C). Altogether, these results show that in parasites at the ring stage neither a DHA pulse nor choline depletion induces sexual conversion, suggesting that this developmental stage is largely insensitive to environmental stimulation of sexual conversion.

\section{CQ exposure at the trophozoite stage can also enhance sexual conversion}

255 Using the same drug pulse approach, we assessed whether $\mathrm{CQ}$, a drug with a different mode of action than DHA (Haldar et al, 2018), also stimulates sexual conversion (Fig. 5A). Exposure to $80 \mathrm{nM} \mathrm{CQ}$ at the trophozoite stage, a dose that induces $\sim 40 \%$ lethality (Fig. 5B), resulted in enhanced sexual conversion rates in choline-supplemented cultures (Fig. 5C-D; Supplementary Fig. 10). However, the level of induction was only $\sim 2$-fold, much lower than induction by DHA, and there was no consistent induction at higher or lower drug doses. Similar to DHA, CQ exposure at the trophozoite stage did not increase sexual conversion in cholinedepleted cultures (Fig. 5C-D), and exposure to CQ at the ring stage did not enhance sexual conversion by either the NCC (Supplementary Fig. 11) or the SCC

265 (Supplementary Fig. 12) routes. Reduced sexual conversion was observed in choline depleted cultures treated with $\mathrm{CQ}$ doses that kill the vast majority of parasites, but this needs to be interpreted with caution because of the intrinsic limitations of sexual 
conversion assays when the majority of parasites are killed (Fig. 5; Supplementary Fig. 10-11).

\section{Enhancement of sexual conversion by DHA operates via pfap2-g}

To determine whether stimulation of sexual conversion by DHA involves activation of the master regulator pfap2-g (PF3D7_1222600), we analysed the transcript levels for this gene after a DHA pulse, and also for one of its earliest known targets, gexp02

275 (Filarsky et al, 2018; Josling et al, 2020; Llorà-Batlle et al, 2020; Portugaliza et al, 2019; Silvestrini et al, 2010). Transcript levels for the two genes were determined at the schizont stage of the cycle of exposure and at the ring stage of the next cycle. $A$ subcurative DHA pulse at the trophozoite stage resulted in upregulation of both pfap2-g and gexp02 relative to the serine-tRNA ligase (PF3D7_0717700) reference gene in choline-supplemented cultures, but not in choline-depleted cultures (Figure $6 \mathrm{~A}-\mathrm{C})$. In contrast, exposure to DHA at the ring stage resulted in reduced expression of both genes (Figure 6D-F). Analysis of transcripts only $2 \mathrm{~h}$ after DHA exposure at the ring stage did not reveal induction of pfap2-g or gexp02 (Fig. 6G-I), ruling out activation of the genes at a time consistent with conversion via the SCC route. Identical results were obtained when normalizing pfap2-g or gexp02 transcript levels against ubiquitin-conjugating (PF3D7_0812600) enzyme as a reference gene (Supplementary Fig. 13). Overall, the findings of these transcriptional analyses clearly mirror the effect of the drug on sexual conversion rates, indicating that induction of sexual conversion by DHA is associated with pfap2- $g$ activation.

\section{DISCUSSION}

ARTs are the key component of ACTs, the most widely used treatment for clinical malaria. Additionally, ACTs may be widely administered in mass drug administration campaigns aimed at malaria elimination. Given that the success of malaria control and elimination efforts largely depends on preventing disease transmission, understanding the impact of ARTs on the production of transmission forms is of paramount importance. Our results show a complex effect of DHA on the trade-off between asexual proliferation and formation of transmission forms. Exposure of 
parasites at the trophozoite stage to subcurative doses of DHA resulted in a large increase in sexual conversion rates and total number of gametocytes, which were viable and infectious to mosquitoes. However, this was not observed when parasites were exposed to the same drug doses at the ring stage. Furthermore, in cultures in which sexual conversion was already stimulated at the metabolic level (i.e., by depletion of choline), DHA did not further stimulate sexual conversion at either stage.

305 The accurate determination of the impact of DHA on sexual conversion rates at different stages was possible thanks to the development of an assay that uses a short drug pulse and reporter parasite lines that enable very early detection of gametocytes by flow cytometry.

The overall effect of a drug on the transmission potential of an infection

310 depends on its effect on the sexual conversion rate, and on several other factors. In the case of ARTs, the stimulation of sexual commitment at the trophozoite stage may not result in an overall increase in transmission due to rapid clearance of asexual parasites, which prevents new rounds of gametocyte production, and to the activity of the drug against developing and mature gametocytes. Indeed, several studies

315 have observed that treatment with drug combinations containing ARTs reduce gametocyte density and the duration of gametocyte carriage (Bousema et al, 2006; Bousema et al, 2010; Ippolito et al, 2017; Karl et al, 2015; Okell et al, 2008; Price et al, 1996; Sawa et al, 2013; Targett et al, 2001; von Seidlein et al, 2001; WWARN_Gametocyte_Study_Group, 2016). Notwithstanding the net reduction of transmission potential commonly observed after ART treatment, it is possible that patients in which many of the parasites are at the trophozoite stage at the time of ART administration may experience a peak of circulating gametocytes $\sim 10$ days after treatment (the time required for gametocyte maturation), if the drug does not kill all parasites. In this regard, it is noteworthy that the largest stimulation of sexual 325 conversion was observed at subcurative doses of the drug. Such low drug concentrations may occur during treatment with substandard or underdosed drugs, through poor compliance with the prescribed regimen, as a consequence of drug malabsorption, or as the drug is eliminated following its natural pharmacokinetics profile. Treatment associated with low ARTs concentration may enable survival of 330 some parasites, and at the same time enhance the probability of sexual conversion. Thus, our findings have potential public health implications for the use of ARTs in 
treatment and elimination strategies. While the benefits of ARTs for malaria treatment clearly outweigh the potential risks, the possibility that ARTs increase the transmission potential of some patients should be taken into account when considering their massive use in preventive treatment or elimination campaigns.

There is ongoing debate regarding whether human malaria parasites can modulate their level of investment in producing transmission forms as a response to "stress" (i.e., a condition that reduces the asexual multiplication rate). Whether the impact of stress on sexual conversion rates is positive (enhancement) or negative (reduction) also remains controversial (Buckling et al, 1999a; Buckling et al, 1999b; Buckling et al, 1997; Koepfli \& Yan, 2018; Peatey et al, 2009; Schneider et al, 2018). Evolutionary theory for life histories predicts that treatment with low doses of antimalarial drugs results in reproductive restraint (reduced sexual conversion) to facilitate within-host survival, whereas treatment with high doses that kill the majority of the parasites elicits terminal investment (increased sexual conversion). The results of a recent study using a murine model of malaria were consistent with this prediction (Schneider et al, 2018). However, our experiments with in vitro cultured $P$. falciparum showed the opposite trend: treatment with low doses of DHA and CQ stimulated sexual conversion, in line with some previous studies using $P$. falciparum 350 (Buckling et al, 1999b; Peatey et al, 2009) or a murine malaria model (Buckling et al, 1999a; Buckling et al, 1997). A possible explanation for the discrepancy with the predictions of evolutionary theory is that in the absence of stress, sexual conversion in $P$. falciparum is already restrained, with estimated conversion rates of $\sim 1 \%$ in human infections (Cao et al, 2019; Eichner et al, 2001). Thus, a further reduction of the investment in transmission upon exposure to low drug doses would not have a substantial impact on within-host survival. Of note, absence of LysoPC and choline, which reduces the multiplication rate of $P$. falciparum cultures and therefore can be considered as another type of sublethal stress signal, also stimulates sexual conversion (Brancucci et al, 2017). Together, the results of experiments with $P$. falciparum cultures exposed to low level of stress do not support the predictions of evolutionary theory, whereas for murine malaria parasites different studies reported conflicting results. In this regard, it is possible that different Plasmodium species use different strategies to adjust sexual conversion rates upon stress: although the role of AP2-G as the master regulator of sexual conversion appears to be widely 
365 conserved in all malaria parasite species, upstream events involved in the regulation of sexual conversion are remarkably different between human and murine parasites. The latter show higher conversion rates, do not alter sexual conversion in response to LysoPC restriction, and their genomes lack a gdv1 ortholog (Ngotho et al, 2019).

Our experiments clearly establish that sexual conversion can be stimulated by 370 exposure to DHA at the trophozoite stage, but not at the early ring stage. Of note, stimulation of sexual conversion by depletion of choline (as a proxy for LysoPC depletion) or by exposure to $C Q$ shows the same stage dependency, suggesting that the ring stage is largely insensitive to stimulation of sexual conversion. At the ring stage, some types of stress, such as exposure to DHA, may induce latency (Barrett 375 et al, 2019; Talman et al, 2019), rather than enhancing sexual conversion. Furthermore, we found that in cultures in which sexual conversion is stimulated by choline depletion, it cannot be further stimulated by drugs, such that there are no additive or synergistic effects between drugs and choline depletion. Together, these observations suggest that the different stimuli converge into the same mechanism of pfap2-g activation, which likely involves cellular components that are absent during the ring stage. Because stimulation of sexual conversion by choline depletion has been shown to involve GDV1 (Filarsky et al, 2018), which is only expressed in the second half of the intraerythrocytic development cycle and is absent from ring stage parasites, we hypothesize that stimulation by DHA may also depend on GDV1. A possible explanation for the similar effects of DHA and choline depletion on sexual conversion is that treatment with DHA may result in choline depletion: DHA induces damage on membrane lipids (Hartwig et al, 2009), which may increase the use of LysoPC or choline, resulting in a reduction of their levels. Alternatively, parasites may be able to sense a state of mild to moderate "stress" or growth restriction 390 (Schneider et al, 2018): the drug doses that result in increased sexual conversion, as well as LysoPC or choline restriction (Brancucci et al, 2017; Portugaliza et al, 2019), are all associated with a $<50 \%$ reduction of multiplication rates (Fig. 1B,E, Fig. $5 B, D)$. In this regard, activation of the cellular stress response has been proposed to be associated with enhanced gametocyte production (Chaubey et al, 2014), and 395 DHA triggers this stress response (Bridgford et al, 2018; Zhang et al, 2017).

Altogether, here we provide a detailed characterization of the changes in $P$. falciparum sexual conversion rates that occur in response to a pulse of DHA. We 
demonstrate remarkable plasticity in sexual conversion rates, and a complex response that depends on the stage of the parasites at the time when they are exposed to the drug, the drug dose, and the metabolic state (presence or absence of choline). This complex scenario may explain the discrepant results obtained by previous studies. The assay that we have developed to test the impact of DHA on sexual conversion rates can be used to test the impact of any other drug or condition. Of note, the success of malaria elimination efforts largely depends on the

405 ability to reduce or interrupt transmission. Although our results are not of immediate public health concern because the overall impact of treatment with ACTs is a reduction of the transmission potential, at least when compared with other drugs, the capacity of ARTs to induce sexual conversion must be taken into account. Otherwise, under certain conditions, treatment may result in an increase in

410 transmission that could jeopardize efforts to eliminate malaria.

\section{METHODS}

\section{Parasite cultures}

The transgenic reporter lines NF54-gexp02-Tom, E5-gexp02-Tom, and NF54-10.3-

415 Tom were previously described and characterized (Portugaliza et al, 2019). These parasite lines carry a tdTomato reporter gene under the control of either the gexp02 or the etramp10.3 promoters. Cultures were regularly maintained at $37^{\circ} \mathrm{C}$ under shaking (100 rpm) or static conditions in a hypoxic atmosphere $\left(2 \% \mathrm{O}_{2}, 5.5 \% \mathrm{CO}_{2}\right.$, balance $\mathrm{N}_{2}$ ), with $\mathrm{B}+$ erythrocytes (3\% hematocrit) and standard RPMI-HEPES parasite culture medium containing 0.5\% Albumax and supplemented with $2 \mathrm{mM}$ choline (Filarsky et al, 2018; Portugaliza et al, 2019). Erythrocytes were obtained from the Catalan official blood bank (Banc de Sang i Teixits). To obtain cultures of a well-defined age window, we used Percoll/sorbitol synchronization. In brief, Percollpurified schizonts were used to establish a fresh culture that 5 or $10 \mathrm{~h}$ later was 425 subjected to $5 \%$ D-sorbitol lysis to obtain cultures of a defined $0-5$ or $0-10 \mathrm{~h}$ post invasion (hpi) age window.

Cultures for the production of mature gametocytes for mosquito infection were maintained in a semi-automated shaker incubator system as described (Mogollon et al, 2016). Fresh human serum and red blood cells (RBC) for these experiments were 
430 obtained from the Dutch National Blood Bank (Sanquin Amsterdam, the Netherlands; permission granted from donors for the use of blood products for malaria research and microbiology; tested for safety). RBC and human serum from different donors were pooled.

\section{Drug treatment and determination of drug survival rates}

To test the impact of drugs on sexual conversion in the presence or absence of choline, after tight synchronization (0-5 or $0-10 \mathrm{hpi}$ ) cultures at $\sim 1.5 \%$ parasitemia were split in two and one culture was maintained with a $2 \mathrm{mM}$ choline supplement whereas the other had no choline added. Drug pulses with DHA (Sigma-Aldrich no.

440 D7439) or CQ (Sigma-Aldrich no. C6628) were performed at 1-6 hpi (NF54-10.3Tom) or 0-10 hpi (NF54-gexp02-Tom) for exposure at the ring stage, or starting at 25-30 hpi for exposure at the trophozoite stage. After $3 \mathrm{~h}$, the drug was removed and fresh pre-warmed culture medium was added. In some experiments, 200 nM DHA was maintained for $48 \mathrm{~h}$ as a 'kill' control (Xie et al, 2014).

The survival rate was calculated as the growth rate in treated cultures divided by the growth rate in control cultures, and expressed as percentage. The growth rate is calculated as the parasitemia at the next cycle after drug exposure (measured at 30-35 hpi or 30-40 hpi) divided by the initial parasitemia (at the cycle of drug exposure, measured before drug treatment). Parasitemia was measured by flow cytometry (see below).

\section{Determination of sexual conversion rates}

We define day 0 (D0) as the first day of the next cycle after drug exposure, which corresponds to the first day of Generation 1 in the schematics in the figures. D1 corresponds to the day when new sexual parasites become stage I gametocytes. When using the NF54-gexp02-Tom and E5-gexp02-Tom lines, the sexual conversion rate was calculated as the sexual stage parasitemia divided by the total (sexual + asexual) parasitemia, and expressed as percentage. Asexual and sexual parasites were quantified by flow cytometry at $~ 30-35$ hpi or $~ 30-40$ hpi (D1) of the cycle after 460 drug treatment, in the absence of chemicals that inhibit asexual replication. When 
using the NF54-10.3-Tom line, the sexual conversion rate was measured by dividing the gametocytemia on day 3 (D3) by the initial ring stage parasitemia on D0. In this case, cultures were treated with $50 \mathrm{mM} \mathrm{N}$-acetyl-d-glucosamine (GlcNAc; SigmaAldrich no. A8625) from D1 onwards to inhibit asexual replication. In experiments with the NF54-10.3-Tom line, gametocytemia was also measured on D0 to identify gametocytes already present in the culture at the beginning of the experiment, but it was found to be negligible. In any case, it was subtracted from D3 gametocytemia, such that only gametocytes newly formed during the assay were considered. In all cases, statistical analysis of differences in sexual conversion was performed using one-way ANOVA with Tukey HSD as the post hoc test. Variance was assumed to be homogenous because the sample size was the same for all groups, and they contain the same type of data.

\section{Flow cytometry}

475 Flow cytometry analysis to measure parasitemia at the cycle of drug exposure was measured using the nucleic acid stain SYTO $11(0.016 \mu \mathrm{M})$ (Life Technologies no. S7573) and a BD FACSCalibur machine as previously described (Rovira-Graells et al, 2016). To measure asexual and tdTomato-positive sexual parasites, we used a BD LSRFortessa ${ }^{\mathrm{TM}}$ machine as previously described (Portugaliza et al, 2019), with small modifications after the addition of the mitochondrial membrane potential MitoTracker ${ }^{\circledR}$ Deep Red FM fluorescent dye (Invitrogen no.M22426) at $0.6 \mu \mathrm{M}$ to identify live parasites (Supplementary Fig. 14 and 15) (Amaratunga et al, 2014). Briefly, the RBC population was defined using the side scatter area (SCC-A) versus the forward scatter area (FSC-A) plot, followed by singlet gating using the forward scatter height (FSC-H) versus FSC-A plot. From the singlet population, the parasites were simultaneously analysed for tdTomato fluorescence (laser: $561 \mathrm{~nm}$; filter: 582/15; power: $50 \mathrm{~mW}$ ), SYTO 11 fluorescence (laser: 488 nm; Filter: 525/50-505LP; power: $50 \mathrm{~mW}$ ), and MitoTracker fluorescence (laser: $640 \mathrm{~nm}$; Filter: 670/14-A; power: $40 \mathrm{~mW}$ ). Total gametocytes were quantified on the double positive gate of the

490 tdTomato versus SYTO 11 plot. Total asexual stages were quantified on the tdTomato-negative but SYTO 11-positive gate, whereas viable asexual stages were 
measured on the tdTomato-negative but MitoTracker-positive gate. Flowing Software version 2.5.1 (Perttu Terho) was used for downstream analysis.

\section{Immunofluorescence assay}

Immunofluorescence assays (IFA) were performed as previously described (Bancells et al, 2019; Portugaliza et al, 2019). Briefly, an aliquot of a culture was treated with 80 nM ML10 (cGMP-dependent protein kinase inhibitor) (Baker et al., 2017), starting at $\sim 30-35$ hpi until $\sim 48-53 \mathrm{hpi}$, to inhibit schizont rupture and allow maturation of

500 gametocytes to the stage when all of them express Pfs16. Air-dried blood smears containing schizonts and stage I gametocytes ( 48-53 hpi) were fixed with 1\% paraformaldehyde in PBS, permeabilised with $0.1 \%$ Triton X-100 in PBS, and blocked with $3 \%$ BSA in PBS. The gametocyte-specific primary antibody mouse-antiPfS16 (1:400; 32F717:B02, a gift from R. Sauerwein, Radboud University) and the goat-anti-mouse IgG-Alexa Fluor 488 secondary antibody (1:1,000, Thermo Fisher no.A11029) were used to identify stage I gametocytes, whereas DAPI (5 $\mu \mathrm{g} / \mathrm{ml})$ was added to stain parasite DNA. IFA slides were mounted using Vectashield (Palex Medical) and viewed under an Olympus IX51 epifluorescence microscope for determination of sexual conversion rates. A minimum of 200 DAPI-positive cells were counted for each sample.

\section{Transcriptional analysis}

Trizol reagent (Invitrogen no. 15596026) was used to collect and preserve total RNA, followed by extraction using a protocol designed for samples with low RNA

515 concentration (Mira-Martínez et al, 2017). Briefly, RNA from Trizol samples was purified using a commercial kit (RNeasy ${ }^{\circledR}$ Mini Kit, Qiagen no. 74104) with additional on-column DNAse treatment (Qiagen no. 79254). Next, cDNA synthesis was performed using the AMV Reverse Transcription System (Promega), with a combination of oligo (dT) and random primers. Quantitative PCR (qPCR) analysis of the cDNA was performed as previously described (Bancells et al, 2019). Transcript levels of pfap2-g and gexp02 were normalized against the housekeeping genes serine-tRNA ligase and ubiquitin-conjugating enzyme. All qPCR primers used have been previously described (Bancells et al, 2019; Portugaliza et al, 2019). Statistical 
analysis of transcript levels was performed using one-way ANOVA with Tukey HSD as the post hoc test, as for the analysis of sexual conversion rates.

\section{Production of mature gametocytes and mosquito feeding}

Cultures maintained in medium containing 0.5\% Albumax and supplemented with 2 $\mathrm{mM}$ choline were synchronised for ring stages by D-Sorbitol treatment and diluted to

530 a final parasitemia of $1.5 \%$. At $22 \mathrm{~h}$ after synchronisation, DHA (5 nM) was added to the cultures for $3 \mathrm{~h}$, and $24 \mathrm{~h}$ later (i.e., after reinvasion) culture conditions were changed to medium with $10 \%$ human serum instead of Albumax and choline, and GlcNac $(50 \mathrm{mM})$ was added to kill asexual stages. GlcNac was maintained for 4 days. Gametocyte cultures were followed during 9-13 days after DHA-treatment with

medium changes twice a day, but without replenishing with fresh RBCs. At days 9-13 gametocyte development was analysed in Giemsa stained blood smears and exflagellation was monitored after activation as described (Marin-Mogollon et al, 2018). Gametocytes (day 11 or 13) were fed to Anopheles stephensi mosquitoes using the standard membrane feeding assay (SMFA) (Marin-Mogollon et al, 2018;

540 Ponnudurai et al, 1989). Oocysts (day 7 and 14) and salivary gland sporozoites (day 14) were counted as described (Marin-Mogollon et al, 2018).

\section{ACKNOWLEDGMENTS}

We are grateful to Robert W. Sauerwein (Radboud University, The Netherlands) for 545 the anti-Pfs16 monoclonal antibody, and to Simon Osborne (LifeArc, UK) and David Baker (LSHTM, UK) for providing the compound ML10 and advice on its use. We thank Oriol Llorà-Batlle (ISGlobal) for help setting gametocyte experiments, Blandine M. Franke-Fayard and Severine Chevalley (Leiden University Medical Center) for support with gametocyte cultures and mosquito infections, and the Flow Cytometry core facility of the IDIBAPS for technical help. This work was supported by a grant from the Spanish Ministry of Economy and Competitiveness (MINECO)/ Agencia Estatal de Investigación (AEI) [SAF2016-76190-R to A.C.], co-funded by the European Regional Development Fund (ERDF, European Union). ITM, UvA and ISGlobal are members of the TransGlobalHealth-Erasmus Mundus Joint Doctorate 
555 Programme, European Union (scholarship number 2016-1346 to H.P.P.). This research is part of ISGlobal's Program on the Molecular Mechanisms of Malaria, which is partially supported by the Fundación Ramón Areces. We acknowledge support from the Spanish Ministry of Science and Innovation through the "Centro de Excelencia Severo Ochoa 2019-2023" Program (CEX2018-000806-S), and support 560 from the Generalitat de Catalunya through the CERCA Program.

\section{COMPETING INTERESTS}

The authors declare no competing interests.

\section{REFERENCES}

Adjalley SH, Johnston GL, Li T, Eastman RT, Ekland EH, Eappen AG, Richman A, Sim BK, Lee MC, Hoffman SL, Fidock DA (2011) Quantitative assessment of Plasmodium falciparum sexual development reveals potent transmission-blocking activity by methylene blue. Proc Natl Acad Sci USA 108: E1214-1223

Amaratunga C, Neal AT, Fairhurst RM (2014) Flow cytometry-based analysis of artemisininresistant Plasmodium falciparum in the ring-stage survival assay. Antimicrob Agents Chemother 58: 4938-4940

Ariey F, Witkowski B, Amaratunga C, Beghain J, Langlois AC, Khim N, Kim S, Duru V, Bouchier C, Ma L, Lim P, Leang R, Duong S, Sreng S, Suon S, Chuor CM, Bout DM, Menard S, Rogers WO, Genton B, Fandeur T, Miotto O, Ringwald P, Le Bras J, Berry A, Barale JC, Fairhurst RM, Benoit-Vical F, Mercereau-Puijalon O, Menard D (2014) A

Babiker HA, Schneider P, Reece SE (2008) Gametocytes: insights gained during a decade of molecular monitoring. Trends Parasitol 24: 525-530

Bancells C, Llora-Batlle O, Poran A, Notzel C, Rovira-Graells N, Elemento O, Kafsack BFC, Cortes A (2019) Revisiting the initial steps of sexual development in the malaria parasite Plasmodium falciparum. Nat Microbiol 4: 144-154 and drug treatment failure. Nat Rev Microbiol 17: 607-620 
Birnbaum J, Scharf S, Schmidt S, Jonscher E, Hoeijmakers WAM, Flemming S, Toenhake CG, Schmitt M, Sabitzki R, Bergmann B, Frohlke U, Mesen-Ramirez P, Blancke Soares A, Herrmann H, Bartfai R, Spielmann T (2020) A Kelch13-defined endocytosis pathway mediates artemisinin resistance in malaria parasites. Science 367: 51-59

Blasco B, Leroy D, Fidock DA (2017) Antimalarial drug resistance: linking Plasmodium falciparum parasite biology to the clinic. Nat Med 23: 917-928

Bousema JT, Schneider P, Gouagna LC, Drakeley CJ, Tostmann A, Houben R, Githure JI, Ord R, Sutherland CJ, Omar SA, Sauerwein RW (2006) Moderate effect of artemisininbased combination therapy on transmission of Plasmodium falciparum. J Infect Dis 193:

$605 \quad 1151-1159$

Bousema T, Drakeley C (2011) Epidemiology and infectivity of Plasmodium falciparum and Plasmodium vivax gametocytes in relation to malaria control and elimination. Clin Microbiol Rev 24: 377-410

Bousema T, Okell L, Shekalaghe S, Griffin JT, Omar S, Sawa P, Sutherland C, Sauerwein R, Ghani AC, Drakeley C (2010) Revisiting the circulation time of Plasmodium falciparum gametocytes: molecular detection methods to estimate the duration of gametocyte carriage and the effect of gametocytocidal drugs. Malar J 9: 136

Brancucci NM, Goldowitz I, Buchholz K, Werling K, Marti M (2015) An assay to probe Plasmodium falciparum growth, transmission stage formation and early gametocyte development. Nat Protoc 10: 1131-1142

620 Brancucci NMB, De Niz M, Straub TJ, Ravel D, Sollelis L, Birren BW, Voss TS, Neafsey DE, Marti M (2018) Probing Plasmodium falciparum sexual commitment at the single-cell level. Wellcome Open Res 3: 70

Brancucci NMB, Gerdt JP, Wang C, De Niz M, Philip N, Adapa SR, Zhang M, Hitz E, 625 Niederwieser I, Boltryk SD, Laffitte MC, Clark MA, Gruring C, Ravel D, Blancke Soares A, Demas A, Bopp S, Rubio-Ruiz B, Conejo-Garcia A, Wirth DF, Gendaszewska-Darmach E, Duraisingh MT, Adams JH, Voss TS, Waters AP, Jiang RHY, Clardy J, Marti M (2017) Lysophosphatidylcholine Regulates Sexual Stage Differentiation in the Human Malaria Parasite Plasmodium falciparum. Cell 171: 1532-1544

Bridgford JL, Xie SC, Cobbold SA, Pasaje CFA, Herrmann S, Yang T, Gillett DL, Dick LR, Ralph SA, Dogovski C, Spillman NJ, Tilley L (2018) Artemisinin kills malaria parasites by damaging proteins and inhibiting the proteasome. Nat Commun 9: 3801

635 Buckling A, Crooks L, Read A (1999a) Plasmodium chabaudi: effect of antimalarial drugs on gametocytogenesis. Exp Parasitol 93: 45-54

Buckling A, Ranford-Cartwright LC, Miles A, Read AF (1999b) Chloroquine increases Plasmodium falciparum gametocytogenesis in vitro. Parasitology 118 ( Pt 4): 339-346 
Buckling AG, Taylor LH, Carlton JM, Read AF (1997) Adaptive changes in Plasmodium transmission strategies following chloroquine chemotherapy. Proc Biol Sci 264: 553-559

Butcher GA (1997) Antimalarial drugs and the mosquito transmission of Plasmodium. Int $J$ Parasitol 27: 975-987

Cao P, Collins KA, Zaloumis S, Wattanakul T, Tarning J, Simpson JA, McCarthy J, McCaw JM (2019) Modeling the dynamics of Plasmodium falciparum gametocytes in humans during malaria infection. Elife 8: e49058

Carter LM, Kafsack BF, Llinas M, Mideo N, Pollitt LC, Reece SE (2013) Stress and sex in malaria parasites: Why does commitment vary? Evol Med Public Health 2013: 135-147

Chaubey S, Grover M, Tatu U (2014) Endoplasmic reticulum stress triggers gametocytogenesis in the malaria parasite. J Biol Chem 289: 16662-16674

Chotivanich K, Sattabongkot J, Udomsangpetch R, Looareesuwan S, Day NP, Coleman RE, White NJ (2006) Transmission-blocking activities of quinine, primaquine, and artesunate. Antimicrob Agents Chemother 50: 1927-1930

de Vries PJ, Dien TK (1996) Clinical pharmacology and therapeutic potential of artemisinin and its derivatives in the treatment of malaria. Drugs 52: 818-836

Delves MJ, Straschil U, Ruecker A, Miguel-Blanco C, Marques S, Baum J, Sinden RE (2016) 665 Routine in vitro culture of $P$. falciparum gametocytes to evaluate novel transmission-blocking interventions. Nat Protoc 11: 1668-1680

Dondorp AM, Nosten F, Yi P, Das D, Phyo AP, Tarning J, Lwin KM, Ariey F, Hanpithakpong W, Lee SJ, Ringwald P, Silamut K, Imwong M, Chotivanich K, Lim P, Herdman T, An SS,

670 Yeung S, Singhasivanon P, Day NP, Lindegardh N, Socheat D, White NJ (2009) Artemisinin resistance in Plasmodium falciparum malaria. N Engl J Med 361: 455-467

Dyer M, Day KP (2000) Commitment to gametocytogenesis in Plasmodium falciparum. Parasitol Today 16: 102-107

Eichner M, Diebner HH, Molineaux L, Collins WE, Jeffery GM, Dietz K (2001) Genesis, sequestration and survival of Plasmodium falciparum gametocytes: parameter estimates from fitting a model to malariatherapy data. Trans $R$ Soc Trop Med Hyg 95: 497-501

680 Filarsky M, Fraschka SA, Niederwieser I, Brancucci NMB, Carrington E, Carrio E, Moes S, Jenoe P, Bartfai R, Voss TS (2018) GDV1 induces sexual commitment of malaria parasites by antagonizing HP1-dependent gene silencing. Science 359: 1259-1263

Fivelman QL, McRobert L, Sharp S, Taylor CJ, Saeed M, Swales CA, Sutherland CJ, Baker 
Haldar K, Bhattacharjee S, Safeukui I (2018) Drug resistance in Plasmodium. Nat Rev Microbiol 16: 156-170

690

Hartwig CL, Rosenthal AS, D'Angelo J, Griffin CE, Posner GH, Cooper RA (2009) Accumulation of artemisinin trioxane derivatives within neutral lipids of Plasmodium falciparum malaria parasites is endoperoxide-dependent. Biochem Pharmacol 77: 322-336

695 Ippolito MM, Johnson J, Mullin C, Mallow C, Morgan N, Wallender E, Li T, Rosenthal PJ (2017) The Relative Effects of Artemether-lumefantrine and Non-artemisinin Antimalarials on Gametocyte Carriage and Transmission of Plasmodium falciparum: A Systematic Review and Meta-analysis. Clin Infect Dis 65: 486-494

700 Josling GA, Russell TJ, Venezia J, Orchard L, van Biljon R, Painter HJ, Llinas M (2020) Dissecting the role of PfAP2-G in malaria gametocytogenesis. Nat Commun 11: 1503

Josling GA, Williamson KC, Llinas M (2018) Regulation of Sexual Commitment and Gametocytogenesis in Malaria Parasites. Annu Rev Microbiol 72: 501-519

Kafsack BF, Rovira-Graells N, Clark TG, Bancells C, Crowley VM, Campino SG, Williams AE, Drought LG, Kwiatkowski DP, Baker DA, Cortes A, Llinas M (2014) A transcriptional switch underlies commitment to sexual development in malaria parasites. Nature 507: 248252

Karl S, Laman M, Moore BR, Benjamin J, Koleala T, Ibam C, Kasian B, Siba PM, Waltmann A, Mueller I, Woodward RC, St Pierre TG, Davis TM (2015) Gametocyte Clearance Kinetics Determined by Quantitative Magnetic Fractionation in Melanesian Children with Uncomplicated Malaria Treated with Artemisinin Combination Therapy. Antimicrob Agents 715 Chemother 59: 4489-4496

Koepfli C, Yan G (2018) Plasmodium Gametocytes in Field Studies: Do We Measure Commitment to Transmission or Detectability? Trends Parasito/ 34: 378-387

720 Llorà-Batlle O, Michel Todó LA, Witmer K, Toda H, Fernández-Becerra C, Baum J, Cortés A (2020) Conditional expression of PfAP2-G for controlled massive sexual conversion in Plasmodium falciparum. Sci Adv 6: eaaz5057

Mairet-Khedim M, Leang R, Marmai C, Khim N, Kim S, Ke S, Kauy C, Kloeung N, Eam R, 725 Chy S, Izac B, Bouth DM, Bustos MD, Ringwald P, Ariey F, Witkowski B (2020) Clinical and in vitro resistance of Plasmodium falciparum to artesunate-amodiaquine in Cambodia. Clin Infect Dis in press, doi: 10.1093/cid/ciaa628

Marin-Mogollon C, van Pul FJA, Miyazaki S, Imai T, Ramesar J, Salman AM, Winkel BMF, 730 Othman AS, Kroeze H, Chevalley-Maurel S, Reyes-Sandoval A, Roestenberg M, FrankeFayard B, Janse CJ, Khan SM (2018) Chimeric Plasmodium falciparum parasites expressing Plasmodium vivax circumsporozoite protein fail to produce salivary gland sporozoites. Malar J 17: 288 
735 McKenzie FE, Bossert WH (1998) The optimal production of gametocytes by Plasmodium falciparum. J Theor Biol 193: 419-428

Mideo N, Day T (2008) On the evolution of reproductive restraint in malaria. Proc Biol Sci 275: $1217-1224$

Mira-Martínez S, van Schuppen E, Amambua-Ngwa A, Bottieau E, Affara M, Van Esbroeck M, Vlieghe E, Guetens P, Rovira-Graells N, Gómez-Perez GP, Alonso PL, D'Alessandro U, Rosanas-Urgell A, Cortés A (2017) Expression of the Plasmodium falciparum Clonally Variant clag3 Genes in Human Infections. J Infect Dis 215: 938-945

Mogollon CM, van Pul FJ, Imai T, Ramesar J, Chevalley-Maurel S, de Roo GM, Veld SA, Kroeze H, Franke-Fayard BM, Janse CJ, Khan SM (2016) Rapid Generation of Marker-Free $P$. falciparum Fluorescent Reporter Lines Using Modified CRISPR/Cas9 Constructs and Selection Protocol. PLOS ONE 11: e0168362

Ngotho P, Soares AB, Hentzschel F, Achcar F, Bertuccini L, Marti M (2019) Revisiting gametocyte biology in malaria parasites. FEMS Microbiol Rev 43: 401-414

Okell LC, Drakeley CJ, Ghani AC, Bousema T, Sutherland CJ (2008) Reduction of transmission from malaria patients by artemisinin combination therapies: a pooled analysis of six randomized trials. Malar J 7: 125

Peatey CL, Skinner-Adams TS, Dixon MW, McCarthy JS, Gardiner DL, Trenholme KR (2009) Effect of antimalarial drugs on Plasmodium falciparum gametocytes. J Infect Dis 200: $1518-1521$

Phyo AP, Ashley EA, Anderson TJC, Bozdech Z, Carrara VI, Sriprawat K, Nair S, White MM, Dziekan J, Ling C, Proux S, Konghahong K, Jeeyapant A, Woodrow CJ, Imwong M, McGready R, Lwin KM, Day NPJ, White NJ, Nosten F (2016) Declining Efficacy of

765 Artemisinin Combination Therapy Against $P$. Falciparum Malaria on the Thai-Myanmar Border (2003-2013): The Role of Parasite Genetic Factors. Clin Infect Dis 63: 784-791

Plouffe DM, Wree M, Du AY, Meister S, Li F, Patra K, Lubar A, Okitsu SL, Flannery EL, Kato N, Tanaseichuk O, Comer E, Zhou B, Kuhen K, Zhou Y, Leroy D, Schreiber SL, Scherer CA, 770 Vinetz J, Winzeler EA (2016) High-Throughput Assay and Discovery of Small Molecules that Interrupt Malaria Transmission. Cell Host Microbe 19: 114-126

Ponnudurai T, Lensen AH, Van Gemert GJ, Bensink MP, Bolmer M, Meuwissen JH (1989) Infectivity of cultured Plasmodium falciparum gametocytes to mosquitoes. Parasitology $98 \mathbf{P t}$ 775 2: $165-173$

Poran A, Notzel C, Aly O, Mencia-Trinchant N, Harris CT, Guzman ML, Hassane DC, Elemento O, Kafsack BFC (2017) Single-cell RNA sequencing reveals a signature of sexual commitment in malaria parasites. Nature 551: 95-99 
Portugaliza HP, Llora-Batlle O, Rosanas-Urgell A, Cortes A (2019) Reporter lines based on the gexp02 promoter enable early quantification of sexual conversion rates in the malaria parasite Plasmodium falciparum. Sci Rep 9: 14595

785 Price RN, Nosten F, Luxemburger C, ter Kuile FO, Paiphun L, Chongsuphajaisiddhi T, White NJ (1996) Effects of artemisinin derivatives on malaria transmissibility. Lancet 347: 16541658

Rajapandi T (2019) Upregulation of gametocytogenesis in anti-malarial drug-resistant Plasmodium falciparum. J Parasit Dis 43: 458-463

Reece SE, Ali E, Schneider P, Babiker HA (2010) Stress, drugs and the evolution of reproductive restraint in malaria parasites. Proc Biol Sci 277: 3123-3129

795 Reece SE, Schneider P (2018) Premature Rejection of Plasticity in Conversion. Trends Parasitol 34: 633-634

Rovira-Graells N, Aguilera-Simon S, Tinto-Font E, Cortes A (2016) New Assays to Characterise Growth-Related Phenotypes of Plasmodium falciparum Reveal Variation in

800 Density-Dependent Growth Inhibition between Parasite Lines. PLoS ONE 11: e0165358

Sawa P, Shekalaghe SA, Drakeley CJ, Sutherland CJ, Mweresa CK, Baidjoe AY, Manjurano A, Kavishe RA, Beshir KB, Yussuf RU, Omar SA, Hermsen CC, Okell L, Schallig HD, Sauerwein RW, Hallett RL, Bousema T (2013) Malaria transmission after artemether-

805 lumefantrine and dihydroartemisinin-piperaquine: a randomized trial. $J$ Infect Dis 207: 16371645

Schneider P, Greischar MA, Birget PLG, Repton C, Mideo N, Reece SE (2018) Adaptive plasticity in the gametocyte conversion rate of malaria parasites. PLoS Pathog 14: e1007371

810

Silvestrini F, Lasonder E, Olivieri A, Camarda G, van Schaijk B, Sanchez M, Younis Younis S, Sauerwein R, Alano P (2010) Protein export marks the early phase of gametocytogenesis of the human malaria parasite Plasmodium falciparum. Mol Cell Proteomics 9: 1437-1448

815 Talman AM, Clain J, Duval R, Menard R, Ariey F (2019) Artemisinin Bioactivity and Resistance in Malaria Parasites. Trends Parasito/ 35: 953-963

Targett G, Drakeley C, Jawara M, von Seidlein L, Coleman R, Deen J, Pinder M, Doherty T, Sutherland C, Walraven G, Milligan P (2001) Artesunate reduces but does not prevent posttreatment transmission of Plasmodium falciparum to Anopheles gambiae. J Infect Dis 183: $1254-1259$

Taylor LH, Read AF (1997) Why so few transmission stages? Reproductive restraint by malaria parasites. Parasitol Today 13: 135-140 
BK, Amoah LE, Williamson KC (2019) Plasmodium falciparum sexual differentiation in malaria patients is associated with host factors and GDV1-dependent genes. Nat Commun 10: 2140

van der Pluijm RW, Imwong M, Chau NH, Hoa NT, Thuy-Nhien NT, Thanh NV, Jittamala P, Hanboonkunupakarn B, Chutasmit K, Saelow C, Runjarern R, Kaewmok W, Tripura R, Peto TJ, Yok S, Suon S, Sreng S, Mao S, Oun S, Yen S, Amaratunga C, Lek D, Huy R, Dhorda

835 M, Chotivanich K, Ashley EA, Mukaka M, Waithira N, Cheah PY, Maude RJ, Amato R, Pearson RD, Goncalves S, Jacob CG, Hamilton WL, Fairhurst RM, Tarning J, Winterberg M, Kwiatkowski DP, Pukrittayakamee S, Hien TT, Day NP, Miotto O, White NJ, Dondorp AM (2019) Determinants of dihydroartemisinin-piperaquine treatment failure in Plasmodium falciparum malaria in Cambodia, Thailand, and Vietnam: a prospective clinical,

840 pharmacological, and genetic study. Lancet Infect Dis 19: 952-961

Venugopal K, Hentzschel F, Valkiūnas G, Marti M (2020) Plasmodium asexual growth and sexual development in the haematopoietic niche of the host. Nat Rev Microbiol 18: 177-189

845 von Seidlein L, Drakeley C, Greenwood B, Walraven G, Targett G (2001) Risk factors for gametocyte carriage in Gambian children. Am J Trop Med Hyg 65: 523-527

World Health Organization (2017) A framework for malaria elimination.

850 WWARN_Gametocyte_Study_Group (2016) Gametocyte carriage in uncomplicated Plasmodium falciparum malaria following treatment with artemisinin combination therapy: a systematic review and meta-analysis of individual patient data. BMC Med 14: 79

Xie SC, Dogovski C, Kenny S, Tilley L, Klonis N (2014) Optimal assay design for

855 determining the in vitro sensitivity of ring stage Plasmodium falciparum to artemisinins. Int $J$ Parasitol 44: 893-899

Yang T, Yeoh LM, Tutor MV, Dixon MW, McMillan PJ, Xie SC, Bridgford JL, Gillett DL, Duffy MF, Ralph SA, McConville MJ, Tilley L, Cobbold SA (2019) Decreased K13 Abundance

860 Reduces Hemoglobin Catabolism and Proteotoxic Stress, Underpinning Artemisinin Resistance. Cell Rep 29: 2917-2928 e2915

Zhang M, Gallego-Delgado J, Fernandez-Arias C, Waters NC, Rodriguez A, Tsuji M, Wek RC, Nussenzweig V, Sullivan WJ, Jr. (2017) Inhibiting the Plasmodium elF2alpha Kinase 865 PK4 Prevents Artemisinin-Induced Latency. Cell Host Microbe 22: 766-776 e764 


\section{FIGURES}
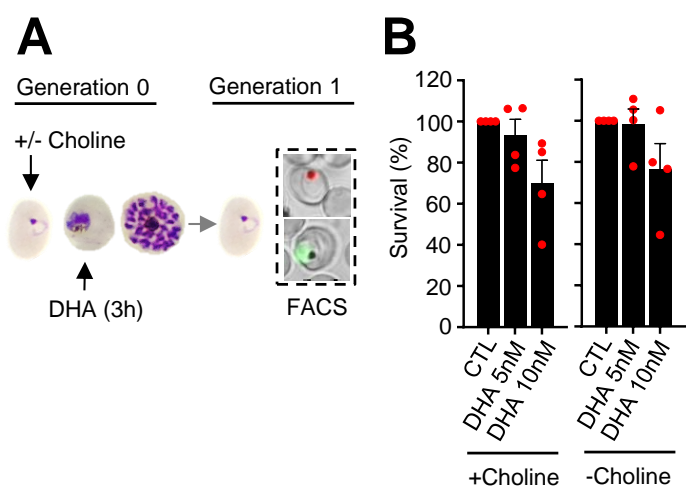

C
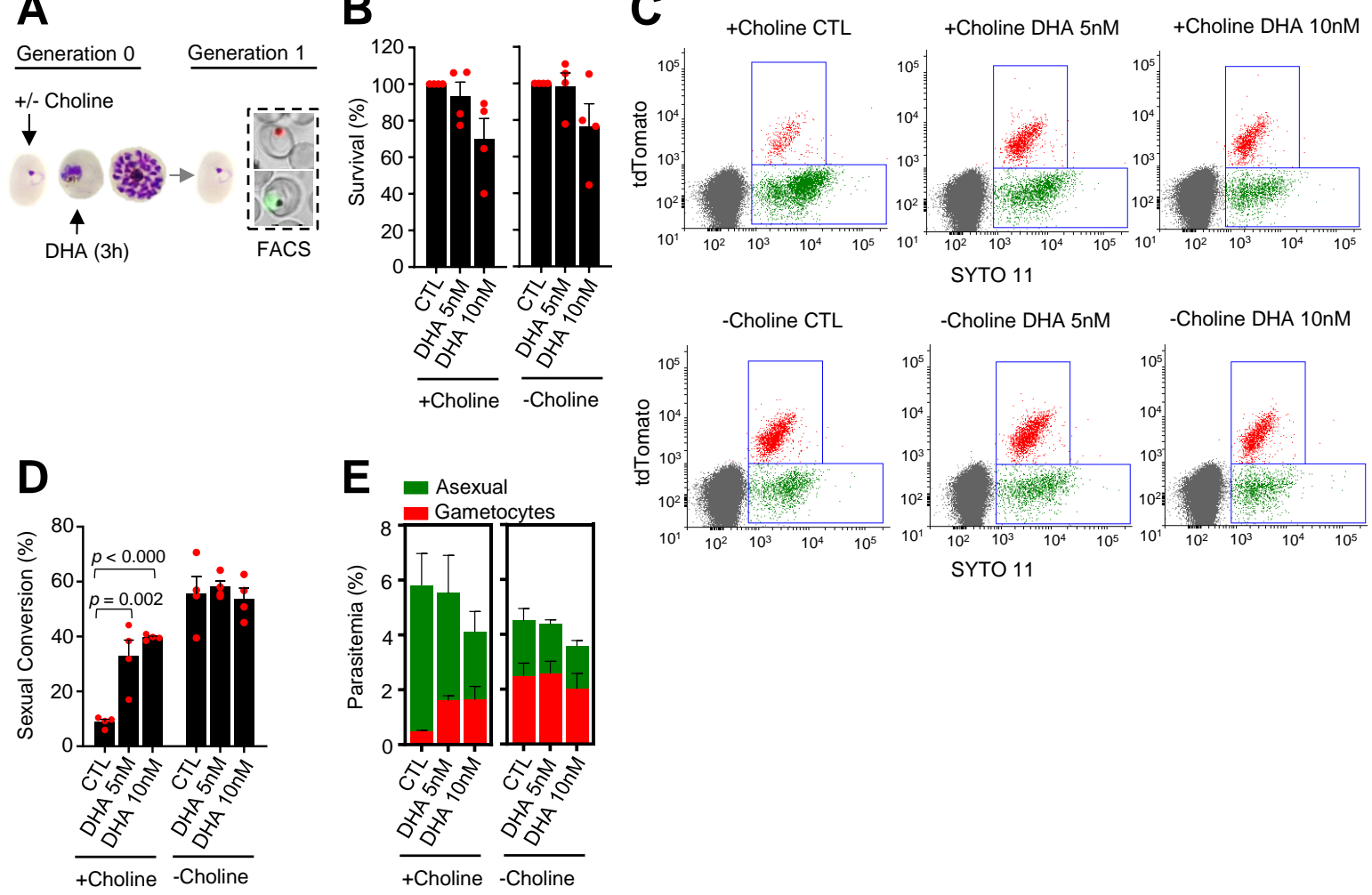

Figure 1. Effect of a dihydroartemisinin (DHA) pulse at the trophozoite stage on sexual conversion. (A) Schematic representation of the assay. Tightly synchronized cultures of the NF54-gexp02-Tom line maintained under non-inducing (+ choline) or inducing (- choline) conditions were exposed to a $3 \mathrm{~h}$ DHA pulse at subcurative doses at the trophozoite stage (25-30 hpi). Sexual conversion was measured by flow cytometry (FACS) after reinvasion ( 30-35 hpi of the next multiplication cycle). (B) Survival rate of cultures exposed to the different drug doses, using total parasitemia values (asexual + sexual parasites). For each choline condition, values are presented relative to the parasitemia in the control cultures (no

880 drug). (C) Representative SYTO 11 (stains parasite DNA) vs TdTomato (marks gametocytes) flow cytometry plots. (D) Sexual conversion rate determined by flow cytometry. The $p$ value is indicated only for treatment vs control (no drug) significant differences $(p<0.05)$. (E) Distribution of absolute parasitemia of asexual and sexual parasites. In all panels, data are presented as the average and s.e.m. of 4 885 independent biological replicates. 

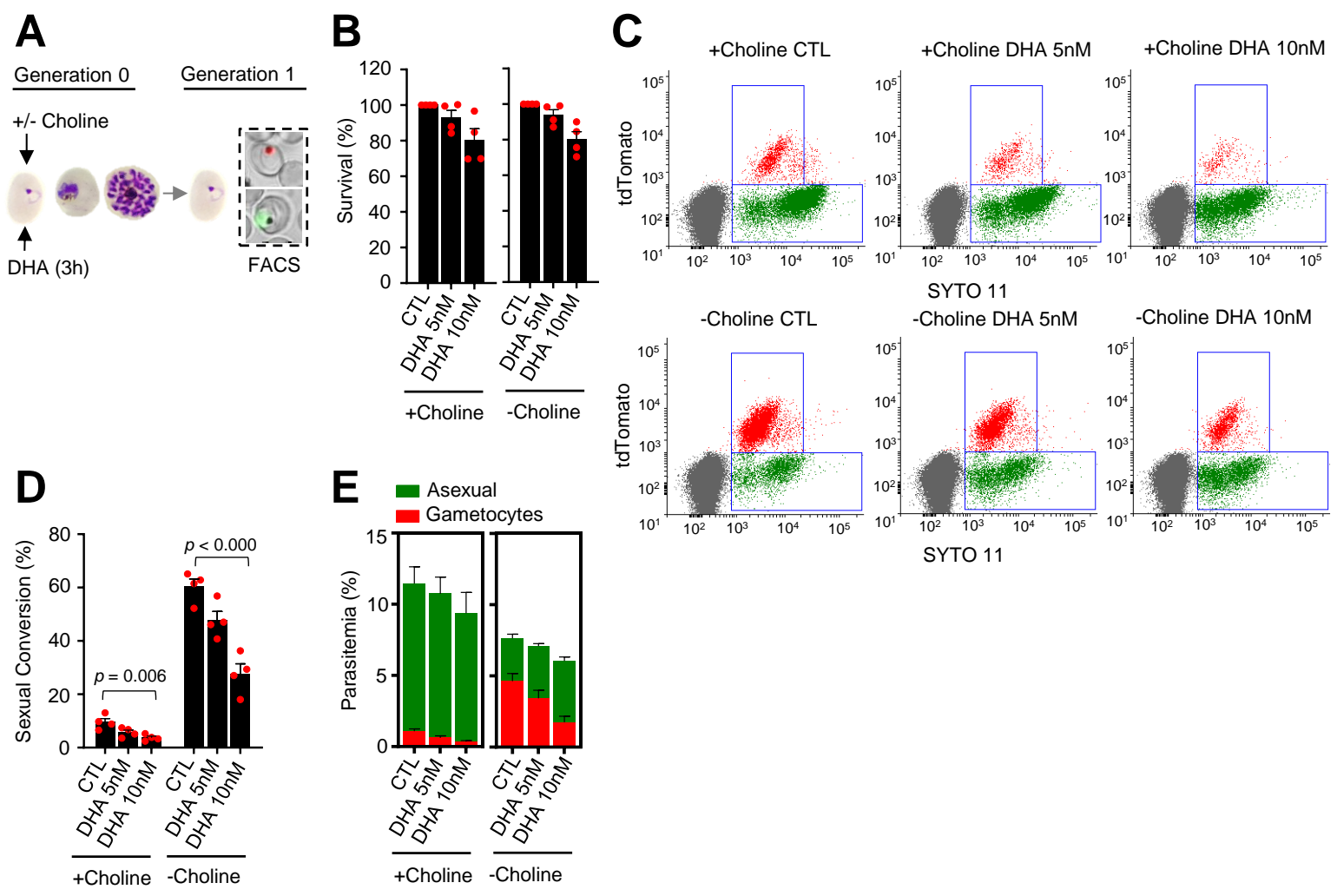

Figure 2. Effect of a dihydroartemisinin (DHA) pulse at the ring stage on sexual conversion. (A) Schematic representation of the assay. Tightly synchronized cultures of the NF54-gexp02-Tom line maintained under non-inducing (+ choline) or inducing (- choline) conditions were exposed to a $3 \mathrm{~h}$ DHA pulse at subcurative doses at the early ring stage (0-10 hpi). Sexual conversion was measured by flow cytometry (FACS) after reinvasion ( 30-40 hpi of the next multiplication cycle). (B) Survival rate of cultures exposed to the different drug doses, using total parasitemia values (asexual + sexual parasites). For each choline condition, values are presented relative to the parasitemia in the control cultures (no drug). (C) Representative SYTO 11 (stains parasite DNA) vs TdTomato (marks gametocytes) flow cytometry plots. (D) Sexual conversion rate determined by flow cytometry. The $p$ value is indicated only for treatment vs control (no drug) significant differences $900 \quad(p<0.05)$. (E) Distribution of absolute parasitemia of asexual and sexual parasites. In all panels, data are presented as the average and s.e.m. of 4 independent biological replicates. 


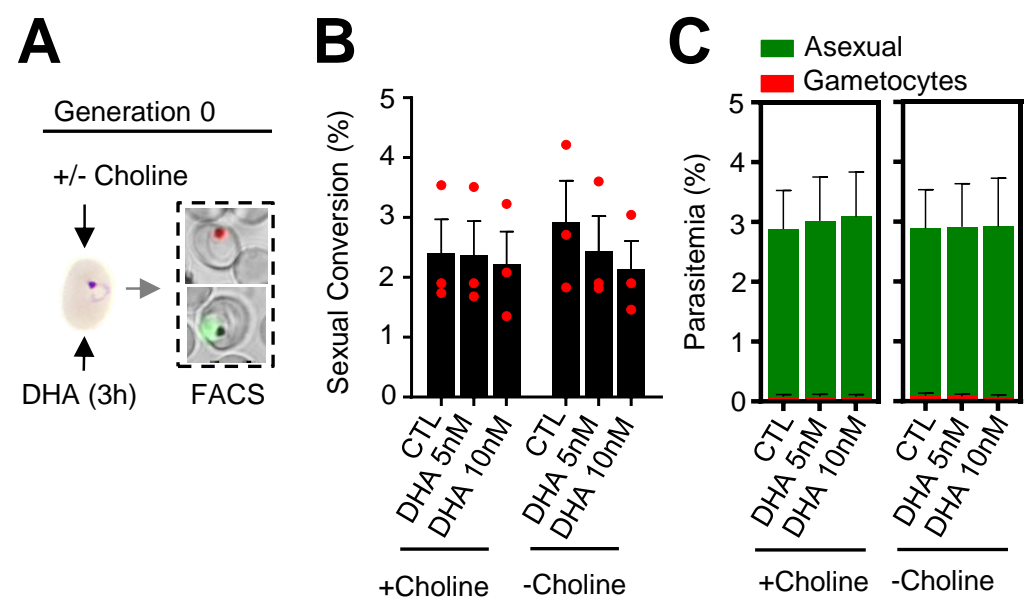

Figure 3. Effect of a dihydroartemisinin (DHA) pulse at the ring stage on sexual conversion by the same cycle conversion (SCC) route. (A) Schematic representation of the assay. Tightly synchronized cultures of the NF54-gexp02-Tom line maintained under non-inducing (+ choline) or inducing (- choline) conditions were exposed to a $3 \mathrm{~h}$ DHA pulse at subcurative doses at the early ring stage (0-10 hpi). Sexual conversion was measured by flow cytometry (FACS) within the same

910 multiplication cycle ( 30-40 hpi) to determine the effect of the drug pulse only on production of new gametocytes by the SSC route. (B) Sexual conversion rate determined by flow cytometry. No significant difference $(p<0.05)$ with the control (no drug) was observed for any treatment condition. (C) Distribution of absolute parasitemia of asexual and sexual parasites. In all panels, data are presented as the 915 average and s.e.m. of 3 independent biological replicates. 

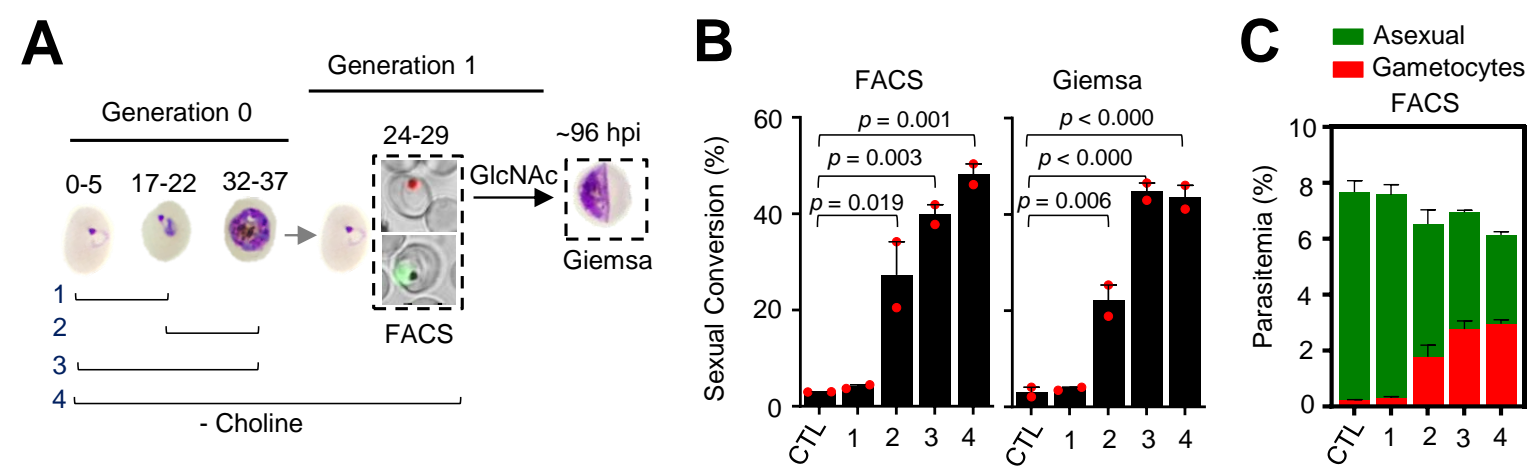

Figure 4. Changes in sexual conversion rates after choline depletion at different parasite stages. (A) Schematic representation of the assay. Choline was removed from tightly synchronized cultures of the NF54-gexp02-Tom line for the periods indicated, and sexual conversion rates measured after reinvasion by flow cytometry (FACS; 24-29 hpi of the following multiplication cycle) or by light microscopy analysis of Giemsa-stained smears (Giemsa; $96 \mathrm{hpi}$ ) in cultures treated with GlcNac. Control (CTL) cultures were maintained with choline all the time. (B)

925 Sexual conversion rate for cultures under different conditions. The $p$ value is indicated only for choline depletion vs control significant differences $(p<0.05)$. (C) Distribution of absolute parasitemia of asexual and sexual parasites, determined by flow cytometry. In all panels, data are presented as the average and s.e.m. of 2 independent biological replicates. 
A

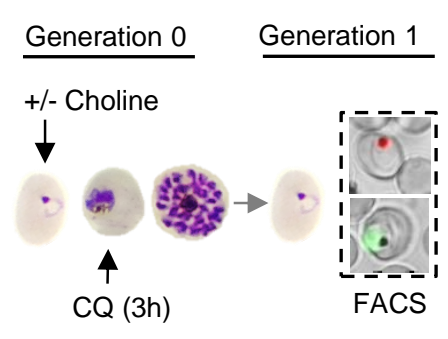

C

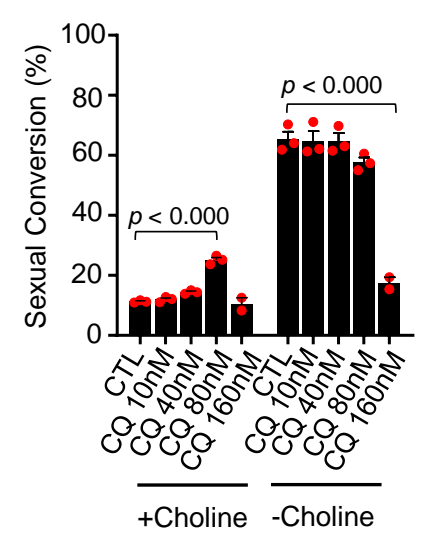

B

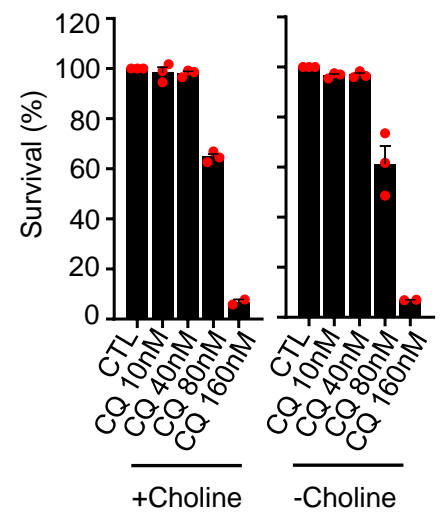

D - Asexual

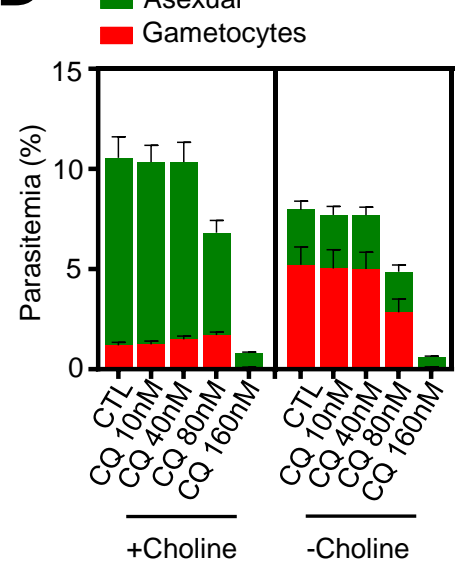

Figure 5. Effect of a chloroquine (CQ) pulse at the trophozoite stage on sexual conversion. (A) Schematic representation of the assay. Tightly synchronized cultures of the NF54-gexp02-Tom line maintained under non-inducing (+ choline) or inducing (- choline) conditions were exposed to a $3 \mathrm{~h} \mathrm{CQ}$ pulse at subcurative doses 935 at the trophozoite stage (25-30 hpi). Sexual conversion was measured by flow cytometry (FACS) after reinvasion ( 30-35 hpi of the next multiplication cycle). (B) Survival rate of cultures exposed to the different drug doses, using total parasitemia values (asexual + sexual parasites). For each choline condition, values are presented relative to the parasitemia in the control cultures (no drug). (C) Sexual conversion rate determined by flow cytometry. The $p$ value is indicated only for treatment vs control (no drug) significant differences $(p<0.05$ ). (D) Distribution of absolute parasitemia of asexual and sexual parasites. In all panels, data are presented as the average and s.e.m. of 3 independent biological replicates. 
A

D

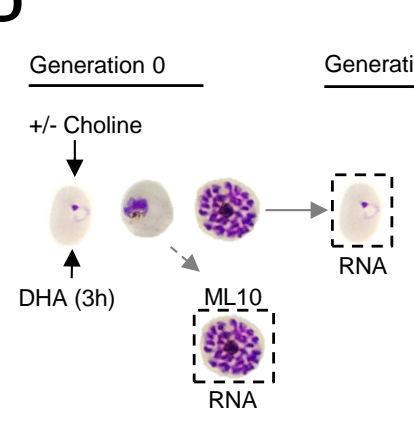

G $\quad$ H

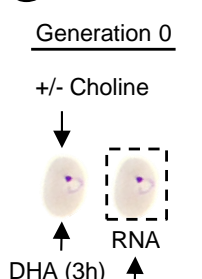

$\mathrm{DHA}(3 \mathrm{~h}) \boldsymbol{4}$

2h Post-DHA
B

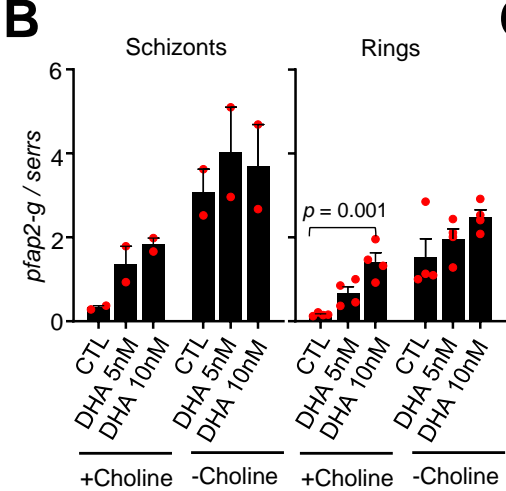

E

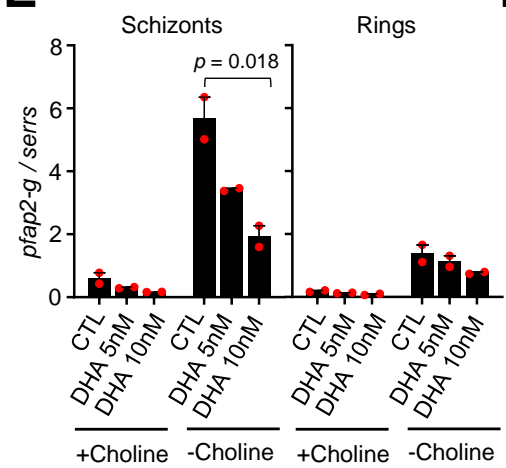

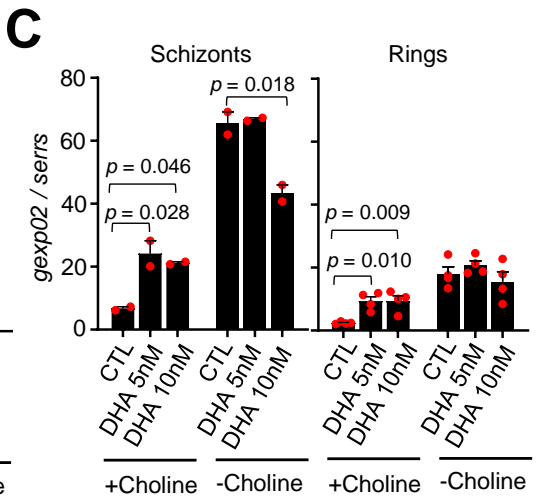

$\mathbf{F}$

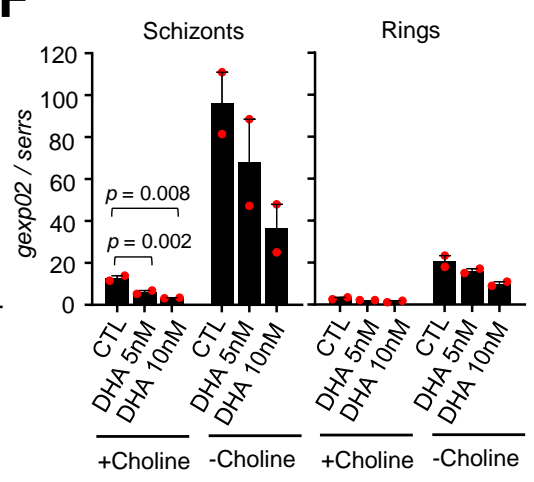

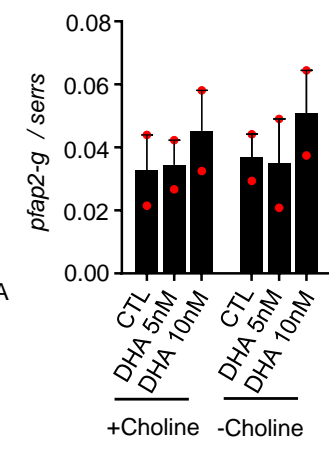

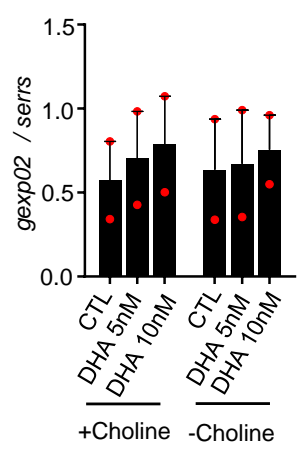

Figure 6. Changes in the expression of pfap2-g and gexp02 after a dihydroartemisinin (DHA) pulse. (A) Schematic representation of the assay. Tightly synchronized cultures of the NF54-gexp02-Tom line maintained under noninducing (+ choline) or inducing (- choline) conditions were exposed to a $3 \mathrm{~h}$ DHA pulse at subcurative doses at the trophozoite stage (25-30 hpi). RNA for transcriptional analysis was collected from ML10-treated cultures at the mature schizont stage (48-53 hpi) and, after reinvasion, from cultures at the early ring stage (cultures not treated with ML10, $\sim$ hpi). (B-C) Transcript levels of pfap2-g (b) or gexp02 (c) normalised against the serine-tRNA ligase (serrs) gene. (D-F) Same as panels A-C, but cultures were exposed to DHA at the ring stage (0-10 hpi). (G-I) Same as panels D-F, but RNA for transcriptional analysis was collected only $2 \mathrm{~h}$ after 
completing the drug pulse. Data are presented as the average and s.e.m. of 4 (panels B-C, rings) or 2 (other panels) independent biological replicates. The $p$ value is indicated only for treatment vs control (no drug) significant differences $(p<0.05)$. 